\title{
Estimation of Combining Ability and Gene Action for Yield and Its Contributing Traits in Bread Wheat (Triticum aestivum.L)
}

Barazan Ahmed Mohammed Al-Mfarji * 1, Prof- Jassim . Mohammed. Aziz Aljubory²

${ }^{1}$ Ministry of Agriculture, Kirkuk Agriculture Directorate . Iraq.

${ }^{2}$ College of Agriculture, Tikrit University. Iraq.

\begin{abstract}
Combining ability analysis for grain yield and its components, and nature of gene action in bread wheat were investigated using twelve lines (L1 "FLORKA" , L2 "MALLIN", L3 "KLAK”, L4 "RIZGARY” ,L5 "ARAS” ,L6 “SITEMALL” ,L7 “HIDHAB” ,L8 “ALLAI” ,L9 “OASIS” ,L10 “KAUZ”, L11 “ADANA99” and L12 "ACSAD5") and three testers (T1 "ABU GHRAIB 3" ,T2 "SHAM 6" and T3 "API 99" ) and thus 36 crosses produced in line $\mathrm{x}$ tester mating design which were evaluated in randomized block design with three replications. Study on gene action revealed the preponderance of non-additive gene action for all traits On the basis of GCA effects, the parents KLAK, ARAS, SITEMALL, ALLAI and tester ABU GHRAIB3 were identified as good general combiners revealing their in desirable direction. Hybrid ABU GHRAIB 3×ALLAI and SHAM6× KAUZ was found to be the best specific cross combination for grain yield and eight traits. However, the best specific cross combinations for other contributing traits were ABU GHRAIB 3× FLORKA for days to 50\% heading, ABU

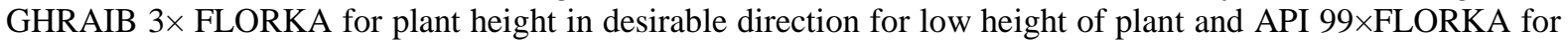
plant height in desirable direction for height of plant, API 99×ADANA 99 and SHAM6× FLORK for Number of spikelets /spike, SHAM6× KAUZ for leaf area ABU GHRAIB $3 \times$ ACSAD5 for number of tillers per plant and Number of spikes /plant, $\times$ ABU GHRAIB 3 ALLAI and API 99×ALLAI Number of grains/spike, API 99×ALLAI Biological yield/plant API 99×ALLAI and ABU GHRAIB $3 \times$ ALLAI for Grain yield/plant, ABU GHRAIB 3 $\times$ ALLAI for harvest index and ABU GHRAIB 3× KLAK for 1000 grain weight.
\end{abstract}

Key words: wheat, line $\times$ tester, combing ability. gene action.

\section{Introduction}

Bread wheat (Triticum aestivum L.) belongs to the family gramineae. It is a self-pollinated, allohexaploid species $(2 n=6 x=42)$, having the genome AABBDD. Therefore, three genomes designated as A, B and D was involved in its evolution (Morris and Sears, 1967). Bread wheat has been cultivated for more than 10,000 years (Poehlman and Sleeper, 1995) and it is the most important food crop of the world and having viscoelastic properties of the endosperm's gluten protein. Wheat is the most extensively cultivated cereal crop and served as the principal food in most areas of the world. Wheat occupies the first place in the world in terms of area where wheat occupies about $32 \%$ of the total area planted with grains globally. The cultivated area (219 million hectares) produced about 771 million tons (FAO ,2018) and the production volume ranges between $45 \%$ and $50 \%$ of the grain production in the Arab world, where it amounts to (2.24077 thousand tons) and constitutes about $3.2 \%$ of the world wheat production, which is about The average wheat production in the Arab world is estimated at (2.97 tons) per hectare for the period 2008-2009 and less than the average global production in 2009 of about 3.5 tons per hectare. $92 \%$ of the total Arab production in 2010, namely Egypt, Morocco, Syria, Algeria, Saudi Arabia and Iraq. Iraq's wheat production in 2018 ranges from 2.2 million tonnes in 2019 to 5.2 million tonnes in 2018, an increase of 81 . (FAO, 2018). At the global level, wheat production is concentrated in the European Union, China, India, the United States of America and the Russian Federation. Buckwheat accounts for about $90-92 \%$ of the world's cultivated wheat (FAO, 2006). This goal can be achieved by enhancing the genetic yield potential of the varieties (Kapoor et al.,2011). To improve yield potential of wheat there is requirement to have knowledge regarding the combining ability of available parents to be used in the hybridization programme and also about the nature of gene action involved in the expression of economically important quantitative as well as qualitative traits (Hassan et al., 2007). The development of genetically superior high yielding varieties, identification of superior parents is an important pre-requisite (Prasad, 2014). Earlier research review revealed that both general and specific combining abilities were involved for yield and yield components (Chaudhry et al., 1992).

The effective improvement in yield of wheat, one can use combining ability analysis to test the performance of selected parents in different cross combinations and can characterise the nature and magnitude of gene effects in the expression of various yield contributing traits. Such information will lead to the selection of superior parental lines and isolation of potential cross combinations for the use in plant breeding programs. Keeping the above in view, the present linextester analysis was planned to estimate general and specific combining ability effects to identify better parents as well as superior cross combinations for further improvement in wheat.

\section{Materials and Methods}

Twelve wheat lines viz., )L1) FLORKA , (L2) MALLIN , (L3) KLAK , (L4) RIZGARY , (L5) ARAS , (L6) SITEMALL , L(7) HIDHAB , (L8) 
ALLAI , (L9) OASIS , (L10) KAUZ , (L11) ADANA99 and (L12) ACSAD5 (were used as females and crossed with three testers viz.,)T1) ABU GHRAIB 3 , (T2) SHAM 6 and (T3) API 99 (in line $\mathrm{x}$ tester fashion. Thirty six $\mathrm{F}_{1 \mathrm{~s}}$ along with fifteen parents were evaluated in randomized complete block design (R.C.B.D) with three replications during 20172018 in timely sown irrigated conditions at in the town of Hawija province of Kirkuk. Parents and $F_{1 s}$ were planted in one rows of three meter length each spaced at $60 \mathrm{~cm}$ and plant to plant distance was maintained at $10 \mathrm{~cm}$ and the observations were recorded on eleven metric traits. Days to 50\% heading (DH), Plant height (PH) , Number of spikelets .spike ${ }^{-1}(\mathrm{NS} / \mathrm{S})$, leaf area (LA), Number of tillers.plant ${ }^{-1}$ (T/P), Number of spikes.plant $^{-1}(\mathrm{NS} / \mathrm{P})$, Number of grains.spike ${ }^{-1}$ (NG/S), Biological yield.plant ${ }^{-1}$ (BY/P), Grain yield.plant $^{-1}(\mathrm{GY} / \mathrm{P}), 1000$ grain weight (TGW), Harvest index (HI). Data were recorded on individual plant basis on a sample of ten randomly selected competitive plants per plot .However, days to $50 \%$ heading were recorded on per plant basis .To test the significance of differences between treatments, analysis of variance was done as suggested by (Panse and Sukhatme ,1967). Combining ability analysis was carried out following the method given by ( Kempthorne ,1957) and later on modified by ( Arunachalam ,1974).

\section{Results and Discussion}

The analysis of variance revealed highly significant differences among treatments for all the characters studied indicating the presence of diversity in the material under study (Table 1). The analysis of variance for combining ability revealed significant variations due to parents, due to parent v/s crosses and due to crosses which is further divided into variation due to lines, testers and line $\times$ tester. all Traits under study (DH), (PH), (NS/S), (LA), (T/P), (NS/P), (NG/S), (BY/P), (GY/P), (TGW), (HI).showed significant variations due to lines as well as testers Variations due to line $\times$ tester interaction components were found significant for all Traits. These findings are in conformity to the earlier reports of (Rajet al., 2013) and (Kapoor et al., 2011). It was observed that the sca variance is greater than the gca variance for all the traits under study (Table 1$)$. The variance ratio $\left(\sigma^{2}\right.$ $\left.\mathrm{gca} / \sigma^{2} \mathrm{sca}\right)$ was found less than one for all traits suggested preponderance of non-additive genetic component in the inheritance of these traits and indicated the importance of these traits in conventional breeding and hybridization techniques for the improvement of wheat in further breeding programmes. Similar findings have been reported by ( Gorjanovic and Balalic ,2004), ( Vanpariya et al., 2006), ( Esmail ,2007), Cifci and Yagdi ,2010) and (Jatav et al., 2014).Dominance genetic variance was found higher than additive variance for all of the traits studied. So it is evident that selection for these traits inherited in this manner should be performed in the later generations like $\mathrm{F}_{4}$ or $\mathrm{F}_{5}$. Fellahi et al.,(2013) .The estimates of gca effects of twelve lines and three testers and sca effects of thirty six F1s for Eleven characters are described and presented in Table 2 (gca) and Table 3 (sca).General combining ability effects for almost all the female lines were found to be significant for most traits, whereas First tester were found to be significant for Days to $50 \%$ heading, Plant height, leaf area, Number of tillers/plant, Number of spikes /plant, 1000 grain weight and The second tester for Plant height, Number of spikelets/spike, Number of spikes /plant, Grain yield/plant and the third tester for leaf area , Number of grains/spike, Harvest index and thousand grains weight. The estimates of general combining ability and specific combining ability effects for different traits are described character wise here as under.

\section{Days to $50 \%$ heading (DH)}

Five parental lines showed significant gca effects. Showed highest significant negative gca effects are desirable by L2 (-2.259) followed by L10 (-1.370), L5 $(-0.703)$, L7 (-0.481) and tester T1 (-0.592). Out of thirty six F1s, Thirteen cross combinations Their values range from $\mathrm{T} 1 \times \mathrm{L} 1(-3.962)$ to $\mathrm{T} 2 \times \mathrm{L} 1, \mathrm{~T} 2 \times$ L5 and T3 $\times$ L4 (-0.740) exhibited significant sca effects in negative direction. Early heading is a very desirable feature for a variety to fit it in different crop rotations thus, negative gca and sca effects are desirable for this trait. L2 was identified as best general combiners for early heading while the cross T1 $\times$ L1 emerged as the best specific combination. Similar significant results have been reported by( Raj et al., 2013) in the F1 generations of line $\times$ tester cross for grain yield and its components in wheat (Triticum aestivum L.).

\section{Plant height (PH)}

Among the parental lines L3 (1.527), L7 (1.527), L8 (1.75), L9 (0.638) L10 (1.861), L12 (0.75) and T2 (0.666) exhibited significant gca effects in positive direction and L1 (-3.361), L5 (-2.027), L6 (-2.138), L1 1 (-0.916) and T1 (-0.555) in negative direction and Twenty-eight cross combinations showed significant sca effects, T1 × L5 (5.666), T1 × L6 (4.111), T1 $\times$ $\mathrm{L} 8$ (1.111), T1 $\times \mathrm{L} 11$ (0.777), T2 $\times$ L2 (4.000), T2 $\times$ L8 (2.555), T2 $\times$ L3 (3.555), T2 $\times$ L12 (2.555), T3 $\times$ L1 (6.555), T3 × L3 (5.333), T3 × L6 (1.000) and T3 $\times \mathrm{L} 7$ (3.000) in positive direction and T1 $\times \mathrm{L} 1(-7.000)$ , T1 × L2 (-1.111), T1 × L3 (-3.888), T1 × L9 $(-0.888)$, $\mathrm{T} 1 \times \mathrm{L} 10(-2.222), \mathrm{T} 2 \times \mathrm{L} 3(-1.444), \mathrm{T} 2 \times \mathrm{L} 5(-3.888)$, T2 $\times$ L6 (-5.111), T2 × L7 (-2.777) T2 × L9 (-1.444) $\mathrm{T} 2 \times \mathrm{L} 11(-1.777) \mathrm{T} 3 \times \mathrm{L} 2(-2.888), \mathrm{T} 3 \times \mathrm{L} 5(-1.777)$, T3 $\times$ L8 (-3.000), T3 $\times$ L11 (-6.333) and T3 $\times$ L12 $(-$ $5.333)$ in negative direction. Dwarf plants are more lodging resistant whereas tall plants are preferred for straw purpose thus preference depends upon the breeding objective. Therefore, L1 can be considered as good general combiner for dwarfness, while L10 
emerged as good general combiner for tallness as these showed significant positive gca effects in negative and positive directions, respectively. Cross $\mathrm{T} 1 \times \mathrm{L} 1$ was identified as the best cross for reduced plant height with high significant. Value of sca effects in negative direction and cross T3 $\times$ L1 was identified for tallness as it showed significant sca effects in positive direction.

Table.1 Analysis of variance for various traits in line $\mathrm{x}$ tester analysis in wheat including parents

\begin{tabular}{|c|c|c|c|c|c|c|c|c|c|c|c|c|}
\hline $\begin{array}{l}\text { Source } \\
\text { of }\end{array}$ & d. & DH & PH & NS/S & LA & $T / P$ & NS/P & NG/S & BY/P & GY/P & TGW & HI \\
\hline $\begin{array}{c}\text { Replica } \\
\text { tions }\end{array}$ & 2 & $\begin{array}{c}117.8 \\
49\end{array}$ & $\begin{array}{c}255.05 \\
8\end{array}$ & $\begin{array}{c}164.1 \\
63\end{array}$ & 3.298 & $\begin{array}{c}289.73 \\
2\end{array}$ & $\begin{array}{c}202.01 \\
9\end{array}$ & $\begin{array}{c}290.47 \\
7\end{array}$ & $\begin{array}{c}49566 . \\
12\end{array}$ & $\begin{array}{c}752.59 \\
4\end{array}$ & $\begin{array}{c}120.02 \\
4\end{array}$ & 0.232 \\
\hline $\begin{array}{c}\text { Genoty } \\
\text { pes }\end{array}$ & $\begin{array}{l}5 \\
0 \\
\end{array}$ & $\begin{array}{c}* * \\
15.99 \\
1 \\
\end{array}$ & $\begin{array}{c}* * \\
53.087\end{array}$ & $\begin{array}{c}* * \\
6.222\end{array}$ & 0.584 & $\begin{array}{c}* * \\
150.62 \\
6 \\
\end{array}$ & 76.069 & $\begin{array}{c}* * \\
330.32 \\
1 \\
\end{array}$ & $\begin{array}{c}* * \\
2182.4 \\
64 \\
\end{array}$ & $\begin{array}{c}* * \\
795.80 \\
5 \\
\end{array}$ & $\begin{array}{c}* * \\
39.724\end{array}$ & 0.018 \\
\hline Parents & $\begin{array}{l}1 \\
4 \\
\end{array}$ & $\begin{array}{c}* * \\
9.99\end{array}$ & $\begin{array}{c}* * * \\
86.561\end{array}$ & $\begin{array}{c}* * \\
7.784\end{array}$ & $\begin{array}{c}* * \\
0.641\end{array}$ & $\begin{array}{c}* * \\
174.85\end{array}$ & $\begin{array}{c}* * \\
73.974\end{array}$ & $\begin{array}{c}* \\
485.75 \\
5 \\
\end{array}$ & $\begin{array}{c}* * \\
1807.5 \\
44 \\
\end{array}$ & $\begin{array}{c}* * \\
847.84 \\
3\end{array}$ & $\begin{array}{c}* * \\
30.137\end{array}$ & $\begin{array}{c}* * \\
0.025\end{array}$ \\
\hline Crosses & $\begin{array}{l}3 \\
5\end{array}$ & $\begin{array}{c}* * \\
17.83\end{array}$ & $\begin{array}{c}* * \\
40.94\end{array}$ & $\begin{array}{c}* * \\
5.742\end{array}$ & $\begin{array}{c}* * \\
0.563\end{array}$ & $\begin{array}{c}* * \\
139.13\end{array}$ & $\begin{array}{c}* * * \\
73.298\end{array}$ & $\begin{array}{c}* * \\
277.53 \\
9\end{array}$ & $\begin{array}{c}* * \\
2333.1 \\
5\end{array}$ & $\begin{array}{c}* * \\
797.25 \\
2\end{array}$ & $\begin{array}{c}* * * \\
44.533\end{array}$ & $\begin{array}{c}* * \\
0.014\end{array}$ \\
\hline $\begin{array}{c}\text { Parents } \\
\text { v/s } \\
\text { Crosses } \\
\end{array}$ & 1 & $\begin{array}{c}* * \\
35.64 \\
\end{array}$ & 9.608 & 1.133 & 0.537 & $\begin{array}{c}* * \\
213.81 \\
2 \\
\end{array}$ & $\begin{array}{c}* * \\
202.37 \\
1 \\
\end{array}$ & 1.594 & $\begin{array}{c}\text { Ns } \\
2157.3 \\
23 \\
\end{array}$ & 16.647 & 5.647 & 0.053 \\
\hline Lines & $\begin{array}{l}1 \\
2\end{array}$ & $\begin{array}{c}* * * \\
11.34\end{array}$ & $\begin{array}{c}* * * \\
27.133\end{array}$ & $\begin{array}{c}* * \\
5.02\end{array}$ & $\begin{array}{c}* * \\
0.749\end{array}$ & $\begin{array}{c}* * \\
181.98 \\
2\end{array}$ & $\begin{array}{c}* * * \\
87.281\end{array}$ & $\begin{array}{c}* * \\
285.46 \\
3\end{array}$ & $\begin{array}{c}* \\
2460.4 \\
5\end{array}$ & $\begin{array}{c}* * \\
1374.7 \\
32\end{array}$ & $\begin{array}{c}* * * \\
70.039\end{array}$ & $\begin{array}{c}* \\
0.012\end{array}$ \\
\hline Testers & 3 & $\begin{array}{c}* * \\
11.25 \\
9 \\
\end{array}$ & $\begin{array}{c}* * * \\
13.777\end{array}$ & $\begin{array}{c}* \\
9.694\end{array}$ & $\begin{array}{c}* * \\
1.277\end{array}$ & $\begin{array}{c}* * \\
126.02 \\
7 \\
\end{array}$ & $\begin{array}{c}* \\
18.064\end{array}$ & $\begin{array}{c}* * \\
491.25 \\
9 \\
\end{array}$ & $\begin{array}{c}* \\
328.18 \\
7 \\
\end{array}$ & $\begin{array}{c}* * \\
97.951\end{array}$ & $\begin{array}{c}* * * \\
48.443\end{array}$ & $\begin{array}{c}* \\
0.005\end{array}$ \\
\hline $\begin{array}{c}\text { Line } \\
\times \text { tester }\end{array}$ & $\begin{array}{l}2 \\
2\end{array}$ & $\begin{array}{c}* * \\
21.67 \\
3\end{array}$ & 50.313 & 5.744 & 0.405 & $\begin{array}{c}* * \\
118.89 \\
6\end{array}$ & 71.327 & $\begin{array}{c}* * \\
254.14 \\
8\end{array}$ & $\begin{array}{c}* * \\
2451.7 \\
69\end{array}$ & $\begin{array}{c}* * \\
572.08 \\
4\end{array}$ & 31.425 & $\begin{array}{c}* * \\
0.016\end{array}$ \\
\hline Error & $\begin{array}{l}\mathbf{1} \\
\mathbf{0} \\
\mathbf{0} \\
\end{array}$ & 1.223 & 1.625 & 1.59 & 0.029 & 1.698 & 1.946 & 2.183 & $\begin{array}{c}632.72 \\
6\end{array}$ & 7.032 & 0.746 & 0.003 \\
\hline \multicolumn{2}{|c|}{$\operatorname{gca}^{2} \sigma$} & $\begin{array}{c}0.059 \\
091\end{array}$ & $\begin{array}{c}0.1371 \\
55\end{array}$ & $\begin{array}{c}0.024 \\
265 \\
\end{array}$ & $\begin{array}{c}0.004 \\
680 \\
\end{array}$ & $\begin{array}{c}1.0039 \\
49\end{array}$ & $\begin{array}{c}0.4367 \\
66 \\
\end{array}$ & $\begin{array}{c}1.8417 \\
59 \\
\end{array}$ & $\begin{array}{c}8.7700 \\
80 \\
\end{array}$ & $\begin{array}{c}6.8495 \\
47 \\
\end{array}$ & $\begin{array}{c}0.3857 \\
96 \\
\end{array}$ & $\begin{array}{c}0.000 \\
049\end{array}$ \\
\hline \multicolumn{2}{|c|}{$\operatorname{sca}^{2} \sigma$} & $\begin{array}{c}6.816 \\
798 \\
\end{array}$ & $\begin{array}{c}16.229 \\
214 \\
\end{array}$ & $\begin{array}{c}1.384 \\
961 \\
\end{array}$ & $\begin{array}{c}0.125 \\
215 \\
\end{array}$ & $\begin{array}{c}39.065 \\
924 \\
\end{array}$ & $\begin{array}{c}23.127 \\
056\end{array}$ & $\begin{array}{c}83.988 \\
119 \\
\end{array}$ & $\begin{array}{c}606.34 \\
7689 \\
\end{array}$ & $\begin{array}{c}188.35 \\
0772 \\
\end{array}$ & $\begin{array}{c}10.226 \\
250 \\
\end{array}$ & $\begin{array}{c}0.004 \\
205 \\
\end{array}$ \\
\hline \multicolumn{2}{|c|}{$\sigma^{2}$ gca/ $\sigma^{2}$ sca } & $\begin{array}{c}0.008 \\
668\end{array}$ & $\begin{array}{c}0.0084 \\
51\end{array}$ & $\begin{array}{c}0.017 \\
520\end{array}$ & $\begin{array}{c}0.037 \\
380\end{array}$ & $\begin{array}{c}0.0256 \\
99\end{array}$ & $\begin{array}{c}0.0188 \\
85\end{array}$ & $\begin{array}{c}0.0219 \\
29\end{array}$ & $\begin{array}{c}0.0144 \\
64\end{array}$ & $\begin{array}{c}0.0363 \\
66\end{array}$ & $\begin{array}{c}0.0377 \\
26\end{array}$ & $\begin{array}{c}0.011 \\
620\end{array}$ \\
\hline \multicolumn{2}{|c|}{$A^{2} \sigma$} & $\begin{array}{c}0.118 \\
183\end{array}$ & $\begin{array}{c}0.2743 \\
11\end{array}$ & $\begin{array}{c}0.048 \\
529\end{array}$ & $\begin{array}{c}0.009 \\
361\end{array}$ & $\begin{array}{c}2.0078 \\
98\end{array}$ & $\begin{array}{c}0.8735 \\
31\end{array}$ & $\begin{array}{c}3.6835 \\
19\end{array}$ & $\begin{array}{c}17.540 \\
160\end{array}$ & $\begin{array}{c}13.699 \\
094\end{array}$ & $\begin{array}{c}0.7715 \\
91\end{array}$ & $\begin{array}{c}0.000 \\
098\end{array}$ \\
\hline \multicolumn{2}{|l|}{$\sigma^{2} D$} & $\begin{array}{c}6.816 \\
798\end{array}$ & $\begin{array}{c}16.229 \\
214\end{array}$ & $\begin{array}{c}1.384 \\
961\end{array}$ & $\begin{array}{c}0.125 \\
215\end{array}$ & $\begin{array}{c}39.065 \\
924\end{array}$ & $\begin{array}{c}23.127 \\
056\end{array}$ & $\begin{array}{c}83.988 \\
119\end{array}$ & $\begin{array}{c}606.34 \\
7689\end{array}$ & $\begin{array}{c}188.35 \\
0772\end{array}$ & $\begin{array}{c}10.226 \\
250\end{array}$ & $\begin{array}{c}0.004 \\
205\end{array}$ \\
\hline \multicolumn{2}{|c|}{$\sigma^{2} \mathbf{A} / \sigma^{2} \mathbf{D}$} & $\begin{array}{c}0.017 \\
337\end{array}$ & $\begin{array}{c}0.0169 \\
02\end{array}$ & $\begin{array}{c}0.035 \\
040\end{array}$ & $\begin{array}{c}0.074 \\
759\end{array}$ & $\begin{array}{c}0.0513 \\
98\end{array}$ & $\begin{array}{c}0.0377 \\
71\end{array}$ & $\begin{array}{c}0.0438 \\
58\end{array}$ & $\begin{array}{c}0.0289 \\
28\end{array}$ & $\begin{array}{c}0.0727 \\
32\end{array}$ & $\begin{array}{c}0.0754 \\
52\end{array}$ & $\begin{array}{c}0.023 \\
240\end{array}$ \\
\hline \multicolumn{2}{|c|}{$\begin{array}{c}\text { Cov H.S. } \\
\text { (line) }\end{array}$} & $\begin{array}{c}1.124 \\
118\end{array}$ & $\begin{array}{c}2.8342 \\
61 \\
\end{array}$ & $\begin{array}{c}0.381 \\
126 \\
\end{array}$ & $\begin{array}{c}0.079 \\
900\end{array}$ & $\begin{array}{c}20.031 \\
514 \\
\end{array}$ & $\begin{array}{c}9.4817 \\
46 \\
\end{array}$ & $\begin{array}{c}31.475 \\
557 \\
\end{array}$ & $\begin{array}{c}203.08 \\
0447\end{array}$ & $\begin{array}{c}151.96 \\
6703 \\
\end{array}$ & $\begin{array}{c}7.6992 \\
82 \\
\end{array}$ & $\begin{array}{c}0.001 \\
061\end{array}$ \\
\hline \multicolumn{2}{|c|}{$\begin{array}{c}\text { Cov H.S. } \\
\text { (tester) }\end{array}$} & $\begin{array}{c}0.278 \\
785\end{array}$ & $\begin{array}{c}0.3375 \\
64\end{array}$ & $\begin{array}{c}0.225 \\
122\end{array}$ & $\begin{array}{c}0.034 \\
647\end{array}$ & $\begin{array}{c}3.4535 \\
86\end{array}$ & $\begin{array}{c}0.4477 \\
37\end{array}$ & $\begin{array}{c}13.585 \\
430\end{array}$ & $\begin{array}{c}- \\
8.4594 \\
02\end{array}$ & $\begin{array}{c}2.5255 \\
40\end{array}$ & $\begin{array}{c}1.3249 \\
26\end{array}$ & $\begin{array}{c}0.000 \\
049\end{array}$ \\
\hline \multicolumn{2}{|c|}{$\begin{array}{l}\text { Cov H.S. } \\
\text { (average) }\end{array}$} & $\begin{array}{c}0.059 \\
091\end{array}$ & $\begin{array}{c}0.1371 \\
55\end{array}$ & $\begin{array}{c}0.024 \\
265\end{array}$ & $\begin{array}{c}0.004 \\
680\end{array}$ & $\begin{array}{c}1.0039 \\
49\end{array}$ & $\begin{array}{c}0.4367 \\
66\end{array}$ & $\begin{array}{c}1.8417 \\
59\end{array}$ & $\begin{array}{c}8.7700 \\
80\end{array}$ & $\begin{array}{c}6.8495 \\
47\end{array}$ & $\begin{array}{c}0.3857 \\
96\end{array}$ & $\begin{array}{c}0.000 \\
049\end{array}$ \\
\hline \multicolumn{2}{|c|}{ Cov F.S. } & $\begin{array}{c}4.334 \\
249 \\
\end{array}$ & $\begin{array}{c}9.1827 \\
87 \\
\end{array}$ & $\begin{array}{c}1.670 \\
473 \\
\end{array}$ & $\begin{array}{c}0.246 \\
185 \\
\end{array}$ & $\begin{array}{c}43.855 \\
986 \\
\end{array}$ & $\begin{array}{c}17.671 \\
416 \\
\end{array}$ & $\begin{array}{c}108.28 \\
8037 \\
\end{array}$ & $\begin{array}{c}345.04 \\
8497 \\
\end{array}$ & $\begin{array}{c}204.30 \\
3812 \\
\end{array}$ & $\begin{array}{c}15.250 \\
350 \\
\end{array}$ & $\begin{array}{c}0.002 \\
514 \\
\end{array}$ \\
\hline
\end{tabular}

$*$, ** significant at $5 \%$ and $1 \%$ levels ,respectively $. \mathrm{DH}=$ Days to $50 \%$ heading, $\mathrm{PH}=\mathrm{Plant}$ height, $\mathrm{NS} / \mathrm{S}=$ Number of spikelets/spike, LA = leaf area, T/P $=$ Number of tillers/plant , NS/P $=$ Number of spikes $/$ plant, NG/S = Number of grains/spike, BY/P= Biological yield/plant, GY/P = Grain yield/plant, TGW=1000 grain weight, $\mathrm{HI}=$ Harvest index 


\section{Number of spikelets per spike (NS/S):}

Three parental lines L1 (1.500), L11 (1.055) and tester T2 $(0.472)$ were found showing significant positive gca effects out of fifteen parental lines and Eleven of the crosses showed significant sca effects for this trait. Viz T1 $\times$ L2 (1.000), T1 $\times$ L4 (1.111), T1 $\times$ L8 (1.888), T1 × L11 (0.888), T1 × L12 (0.888), T2 $\times \mathrm{L} 1$ (2.527), T2 $\times$ L10 (1.861), T3 × L5 (0.805), T3 $\times$ L8 (1.250), T3 $\times$ L9 (1.916) and T3 $\times$ L1 1 (3.583) number of spikelets per spike is an important yield contributing trait. Selection for good general combiners for spikelets per spike includes parents with high positive gca effects. Thus, L1 was identified as best general combiner and T3 $\times$ L11 was identified as best Sca for the trait. Cifci and Yagdi (2010) found similar findings in their F1 and F2 crosses that have been obtained by line $\times$ tester mating. (Zeeshan et al., 2013) and (Kumar et al., 2015) had also reported the similar type of results in their experiment.

Table 2. Estimates of general combining ability effects of parents for different traits

\begin{tabular}{|c|c|c|c|c|c|c|c|c|c|c|c|c|}
\hline S.No. & Genotypes & DH & PH & NS/S & LA & $T / P$ & NS/P & NG/S & BY/P & GY/P & TGW & HI \\
\hline 1 & FLORKA & 1.629 & -3.361 & 1.5 & 0.298 & 2.805 & -0.546 & -1.046 & $\begin{array}{c}- \\
25.311\end{array}$ & $\begin{array}{c}- \\
22.701\end{array}$ & $\begin{array}{c}- \\
3.124\end{array}$ & -0.071 \\
\hline 2 & MALLIN & $\begin{array}{c}- \\
2.259\end{array}$ & 0.416 & 0.388 & $\begin{array}{c}- \\
0.348\end{array}$ & -6.416 & -2.657 & 5.064 & 9.720 & -6.669 & 0.793 & 0.018 \\
\hline 3 & KLAK & $\begin{array}{c}- \\
0.259\end{array}$ & 1.527 & 0.388 & 0.236 & 2.916 & 4.342 & 3.731 & 12.897 & 9.215 & 5.488 & 0.010 \\
\hline 4 & RIZGARY & 0.074 & -0.027 & -1.055 & 0.347 & 1.027 & -0.101 & -8.712 & -0.678 & -1.858 & 3.934 & -0.009 \\
\hline 5 & ARAS & $\begin{array}{c}- \\
0.703\end{array}$ & -2.027 & -0.722 & 0.201 & -2.972 & -1.435 & 0.842 & 20.219 & 14.498 & $\begin{array}{c}- \\
0.538\end{array}$ & 0.042 \\
\hline 6 & SITE MALL & $\begin{array}{c}- \\
0.037\end{array}$ & -2.138 & -0.5 & 0.228 & -2.861 & -0.435 & 7.398 & 8.629 & 13.228 & 2.154 & 0.052 \\
\hline 7 & HIDHAB & $\begin{array}{c}- \\
0.481\end{array}$ & 1.527 & -0.5 & $\begin{array}{c}- \\
0.276\end{array}$ & -6.638 & -6.435 & -6.268 & $\begin{array}{c}- \\
22.109\end{array}$ & -13.35 & $\begin{array}{c}- \\
1.279\end{array}$ & -0.047 \\
\hline 8 & ALLAI & 1.407 & 1.75 & 0.166 & $\begin{array}{c}- \\
0.123\end{array}$ & 7.25 & 1.564 & 9.287 & 23.607 & 18.182 & $-\overline{-}$ & 0.034 \\
\hline 9 & OASIS & 0.740 & 0.638 & -0.388 & 0.283 & -1.527 & -0.768 & -1.824 & -3.496 & -5.752 & $\begin{array}{c}- \\
2.171\end{array}$ & -0.040 \\
\hline 10 & KAUZ & $\begin{array}{c}- \\
1.370 \\
\end{array}$ & 1.861 & 0.055 & $\begin{array}{c}- \\
0.168\end{array}$ & -2.194 & 0.453 & -1.712 & 0.179 & 0.918 & $\begin{array}{c}-\overline{-} \\
3.429 \\
\end{array}$ & 0.022 \\
\hline 11 & ADANA 99 & 0.851 & -0.916 & 1.055 & $\begin{array}{c}- \\
0.296\end{array}$ & 2.583 & 0.231 & -0.046 & 0.082 & 3.768 & $\begin{array}{c}- \\
2.296\end{array}$ & -0.010 \\
\hline 12 & ACSAD5 & 0.407 & 0.75 & -0.388 & $\begin{array}{c}- \\
0.382\end{array}$ & 6.027 & 5.787 & -6.712 & $\begin{array}{c}- \\
23.739\end{array}$ & -9.479 & 1.004 & -0.001 \\
\hline 13 & ABU GHRAIB 3 & $\begin{array}{c}- \\
0.592\end{array}$ & -0.555 & -0.555 & 0.143 & 1.972 & 0.703 & -0.518 & -2.445 & -1.033 & 1.034 & -0.012 \\
\hline 14 & SHAM 6 & 0.518 & 0.666 & 0.472 & $\begin{array}{c}- \\
0.213 \\
\end{array}$ & -1.75 & 0.009 & -3.407 & -0.928 & 1.902 & $\begin{array}{c}- \\
1.253 \\
\end{array}$ & 0.001 \\
\hline 15 & API 99 & 0.074 & -0.111 & 0.083 & 0.069 & -0.222 & -0.712 & 3.925 & 3.374 & -0.868 & 0.219 & 0.010 \\
\hline & SE(LINE) & 0.368 & 0.424 & 0.420 & 0.057 & 0.434 & 0.465 & 0.492 & 8.384 & 0.883 & 0.287 & 0.019 \\
\hline & SE(TESTER) & 0.184 & 0.212 & 0.210 & 0.028 & 0.217 & 0.232 & 0.246 & 4.192 & 0.441 & 0.143 & 0.009 \\
\hline
\end{tabular}

$\mathrm{DH}=$ Days to $50 \%$ heading, $\mathrm{PH}=$ Plant height, $\mathrm{NS} / \mathrm{S}=$ Number of spikelets/spike, $\mathrm{LA}=$ leaf area, $\mathrm{T} / \mathrm{P}=\mathrm{Number}$ of tillers/plant, $\mathrm{NS} / \mathrm{P}=$ Number of spikes /plant, NG/S $=$ Number of grains/spike, BY/P= Biological yield/plant, GY/P = Grain yield/plant, TGW=1000 grain weight, $\mathrm{HI}=$ Harvest index .

\section{Leaf area (LA) :}

For leaf area L1 (0.298), L3 (0.236), L4 (0.347), L5 (0.201), L6 (0.228), L9 (0.283), T1 (0.143) and T3 (0.069) exhibited significant positive gca effects and while Fourteen of the cross combinations showed significant sca effects for the trait. Leaf area is responsible for more than $70 \%$ photosynthesis and thus is very important for grain filling. Thus, L4 (0.347) identified as best general combiner with highest significant positive gca effects and T2 $\times$ L10 was found to be the best specific cross combination for leaf area. ( Shabbir et al., 2012) also observed similar type of results in their line $\times$ tester experiment in bread wheat.

Number of tillers per plant (T/P):

Out of fifteen parents only seven parental line L1 (2.805) , L3 (2.916), L4 (1.027), L8 (7.250) , L11 (2.533) , L12 (6.027) and T1 (1.972) showed significant positive gca effects. twenty three cross combinations exhibited significant sca effects in positive direction and Their values ranged from $\mathrm{T} 1 \times$ L12 (23.694) to T3 $\times$ L5 (1.222). Therefore, parental line L8 identified as best general combiner, also T1 $\times$ 
L12, T1 $\times$ L8 and T3 $\times$ L8 as best cross combinations and these can be used for increased number of tillers per plant. The results showed similarity with the earlier work of (Kumar et al., 2015) using 21 wheat hybrids developed in a half diallel mating design.

Number of spikes per plant (NS/P) :

Three female lines out of twelve exhibited significant gca effects, three lines namely L12 (5.787), L3 (4.342) and L8 (1.564) showed significant positive gca effects for the trait. One tester T1 (0.703) showed significant positive gca effects. twenty two hybrids showed significant sca effects out of which twelve crosses namely T1 $\times$ L12 (18.185), T3 × L8 (17.601), $\mathrm{T} 3 \times \mathrm{L} 12(12.268), \mathrm{T} 2 \times \mathrm{L} 10(11.546), \mathrm{T} 2 \times \mathrm{L} 11$ (10.212), T1 × L3 (9.407), T3 × L11 (6.268), T2 × L8 : T2 $\times$ L12 (6.212), T2 × L9 (5.879), T1 × L9 (5.851), T3 $\times$ L9 : T3 $\times$ L10 (5.268), T1 $\times$ L10 (3.851), T2 $\times$ $\mathrm{L} 2$ (3.768), T1 $\times \mathrm{L} 11$ (3.518), T1 $\times$ L6 (3.185), T2 $\times$ $\mathrm{L} 7$ (2.546), T2 $\times$ L5 (1.879), T3 $\times$ L1 (1.712), T1 $\times$ L2 (1.407) and T3 $\times$ L4 (0. 935) .

Table 3. Estimates of specific combining ability effects of crosses for different traits

\begin{tabular}{|c|c|c|c|c|c|c|c|c|c|c|c|c|}
\hline S.No. & Genotypes & DH & PH & NS/S & LA & $\mathbf{T} / \mathbf{P}$ & NS/P & NG/S & BY/P & GY/P & TGW & HI \\
\hline 1 & $\begin{array}{l}\text { ABU GHRAIB 3× } \\
\text { FLORKA }\end{array}$ & $\begin{array}{c}- \\
3.962\end{array}$ & -7 & $1 . \overline{444}$ & 0.290 & -9.416 & -2.370 & 0.962 & -0.274 & 11.926 & -1.392 & 0.070 \\
\hline 2 & ABU GHRAIB 3× MALLIN & 0.259 & $\begin{array}{c}- \\
1.111\end{array}$ & 1 & $-\overline{1}$ & 2.138 & 1.407 & 14.185 & 22.440 & 3.596 & 3.163 & 0.002 \\
\hline 3 & ABU GHRAIB $3 \times$ KLAK & 1.592 & $\begin{array}{c}- \\
3.888\end{array}$ & 0 & 0.258 & 7.472 & 9.407 & 16.148 & 11.422 & 9.813 & 7.004 & 0.047 \\
\hline 4 & ABU GHRAIB3 $\times$ RIZGARY & 2.925 & $\overline{-}$ & 1.111 & 0.508 & -0.638 & -0.814 & 4.296 & -5.075 & 7.247 & 2.395 & 0.053 \\
\hline 5 & ABU GHRAIB $3 \times$ ARAS & 0.703 & 5.666 & 0.444 & $\begin{array}{c}- \\
0.372\end{array}$ & -3.638 & -1.814 & 1.740 & $\begin{array}{c}- \\
10.179\end{array}$ & -9.715 & -0.581 & -0.052 \\
\hline 6 & ABUGHRAIB3×SITEMALL & 0.370 & 4.111 & $-\overline{111}$ & $\begin{array}{c}- \\
0.674\end{array}$ & 1.916 & 3.185 & 2.518 & 17.538 & 8.495 & -1.321 & 0.032 \\
\hline 7 & ABU GHRAIB $3 \times$ HIDHAB & 1.481 & $\begin{array}{c}- \\
0.222 \\
\end{array}$ & - & 0.028 & -5.638 & -1.148 & -6.148 & $\begin{array}{c}- \\
11.299 \\
\end{array}$ & $\begin{array}{c}- \\
23.108\end{array}$ & -1.620 & -0.133 \\
\hline 8 & ABU GHRAIB $3 \times$ ALLAI & 0.481 & 1.111 & 1.888 & 0.549 & 16.694 & 0.185 & 24.518 & 34.894 & 31.638 & 0.596 & 0.146 \\
\hline 9 & ABU GHRAIB $3 \times$ OASIS & $\begin{array}{c}- \\
1.518\end{array}$ & $\begin{array}{c}- \\
0.888\end{array}$ & - & 0.618 & 3.361 & 5.851 & 1.518 & -3.496 & 0.367 & -4.178 & 0.030 \\
\hline 10 & ABU GHRAIB $3 \times$ KAUZ & $\begin{array}{c}- \\
1.185\end{array}$ & $2 . \overline{2} 22$ & 0.222 & $\begin{array}{c}- \\
0.209\end{array}$ & 0.694 & 3.851 & -0.481 & 34.789 & 2.427 & -6.973 & $\begin{array}{c}- \\
0.0423\end{array}$ \\
\hline 11 & $\begin{array}{l}\text { ABU GHRAIB } 3 \\
\text { ×ADANA99 }\end{array}$ & 1.481 & 0.777 & 0.888 & $\begin{array}{c}- \\
0.082\end{array}$ & 8.694 & 3.518 & 5.518 & 47.476 & 19.122 & -1.308 & 0.004 \\
\hline 12 & ABU GHRAIB $3 \times$ ACSAD5 & 1.814 & 0.444 & 0.888 & $\begin{array}{c}- \\
0.105 \\
\end{array}$ & 23.694 & 18.185 & -2.148 & 13.825 & 12.576 & 3.190 & 0.0788 \\
\hline 13 & SHAM6× FLORK & $\begin{array}{c}- \\
0.740\end{array}$ & 0.444 & 2.527 & $\begin{array}{c}- \\
0.147 \\
\end{array}$ & -5.027 & 0.657 & 4.185 & $\begin{array}{c}- \\
32.707 \\
\end{array}$ & -8.285 & 0.137 & 0.031 \\
\hline 14 & SHAM6 $\times$ MALLIN & 1.148 & 4 & 0.305 & 0.103 & 4.861 & 3.768 & 3.074 & 5.838 & 16.037 & 0.689 & -0.018 \\
\hline 15 & SHAM6×KLAK & $\begin{array}{c}- \\
0.518\end{array}$ & $1 . \overline{4}$ & $\begin{array}{c}- \\
0.694 \\
\end{array}$ & 0.277 & -4.472 & -7.898 & 11.740 & $\begin{array}{c}- \\
23.467 \\
\end{array}$ & $\begin{array}{c}- \\
11.998 \\
\end{array}$ & -4.379 & -0.003 \\
\hline 16 & SHAM6× RIZGAR & $\begin{array}{c}- \\
2.185\end{array}$ & - & 0.416 & $\begin{array}{c}- \\
0.553\end{array}$ & 1.416 & -0.120 & -1.481 & 33.861 & -0.767 & -0.948 & -0.075 \\
\hline 17 & SHAM6 $\times$ ARAS & $0 . \overline{740}$ & $\begin{array}{c}- \\
3.888\end{array}$ & -1.25 & - & 2.416 & 1.879 & -1.370 & 5.689 & 0.930 & 2.514 & -0.028 \\
\hline 18 & SHAM6×SITEMALL & $\begin{array}{c}- \\
0.074\end{array}$ & 5.111 & 0.194 & 0.244 & -1.027 & -1.453 & -4.259 & 22.540 & -5.152 & -3.308 & 0.048 \\
\hline 19 & SHAM6× HIDHAB & $\begin{array}{c}- \\
0.296\end{array}$ & $\begin{array}{c}- \\
2.777\end{array}$ & 0.194 & $\begin{array}{c}- \\
0.102\end{array}$ & 0.75 & 2.546 & -2.925 & 27.293 & 19.222 & -0.359 & 0.057 \\
\hline 20 & SHAM6 $\times$ ALLAI & $\begin{array}{c}- \\
0.296\end{array}$ & 2.555 & $\begin{array}{c}- \\
1.138\end{array}$ & 0.011 & 9.083 & 6.212 & -1.259 & 11.632 & 14.556 & 2.498 & 0.081 \\
\hline 21 & SHAM6 $\times$ OASIS & 5.037 & $\begin{array}{c}- \\
1.444 \\
\end{array}$ & 0.194 & 0.432 & 9.416 & 5.879 & 0.074 & 73.432 & 24.850 & 2.381 & -0.016 \\
\hline 22 & SHAM6 $\times$ KAUZ & 1.703 & 3.555 & 1.861 & 0.665 & 9.75 & 11.546 & 15.407 & 30.889 & 24.405 & 0.441 & 0.125 \\
\hline 23 & SHAM6× ADANA 99 & $\begin{array}{c}- \\
1.962\end{array}$ & $\begin{array}{c}- \\
1.777\end{array}$ & 0.194 & 0.229 & 11.75 & 10.212 & 4.740 & 15.075 & 14.904 & -1.429 & 0.072 \\
\hline 24 & SHAM6 $\times$ ACSAD5 & 3.370 & 2.555 & 0.194 & 0.196 & 6.416 & 6.212 & 2.407 & $\begin{array}{c}- \\
17.812\end{array}$ & 14.315 & 0.735 & -0.035 \\
\hline 25 & API 99×FLORKA & 4.703 & 6.555 & $\begin{array}{c}- \\
1.083\end{array}$ & $\begin{array}{c}- \\
0.143\end{array}$ & 14.444 & 1.712 & -5.148 & 32.982 & -3.641 & 1.254 & -0.101 \\
\hline 26 & API 99×MALLIN & $\begin{array}{c}- \\
1.407\end{array}$ & 2.888 & $\begin{array}{c}- \\
1.305\end{array}$ & 0.008 & -7 & -5.175 & $\begin{array}{c}- \\
17.259\end{array}$ & 16.602 & $\begin{array}{c}- \\
19.633 \\
\end{array}$ & -3.852 & 0.015 \\
\hline 27 & API 99×KLAK & $\begin{array}{c}- \\
1.074\end{array}$ & 5.333 & 0.694 & 0.018 & -3 & -1.509 & 4.407 & 12.045 & 2.185 & -2.624 & -0.044 \\
\hline 28 & API 99×RIZGARY & $\begin{array}{c}- \\
0.740\end{array}$ & 0.555 & $\begin{array}{c}- \\
1.527\end{array}$ & 0.045 & -0.777 & 0.935 & -2.814 & $\begin{array}{c}- \\
28.785\end{array}$ & -6.479 & -1.446 & 0.021 \\
\hline 29 & API 99×ARAS & 0.037 & $\begin{array}{c}- \\
1.777\end{array}$ & 0.805 & 0.477 & 1.222 & -0.064 & -0.370 & 4.489 & 8.784 & -1.932 & 0.080 \\
\hline 30 & API 99×SITE MALL & $\begin{array}{c}- \\
0.296\end{array}$ & 1 & $\begin{array}{c}- \\
0.083\end{array}$ & 0.429 & -0.888 & -1.731 & 1.740 & 5.002 & -3.342 & 4.629 & -0.080 \\
\hline 31 & API 99×HIDHAB & $\begin{array}{c}- \\
1.185\end{array}$ & 3 & $\begin{array}{c}- \\
0.083\end{array}$ & 0.074 & 4.888 & -1.398 & 9.074 & $\begin{array}{c}- \\
15.993\end{array}$ & 3.886 & 1.979 & 0.076 \\
\hline 32 & API 99×ALLAI & 5.481 & -3 & 1.25 & $\begin{array}{c}- \\
0.101\end{array}$ & 15.888 & 17.601 & 23.407 & 90.624 & 48.402 & -0.856 & 0.015 \\
\hline
\end{tabular}




\begin{tabular}{|c|c|c|c|c|c|c|c|c|c|c|c|c|}
\hline 33 & API 99×OASIS & 0.148 & $\begin{array}{c}- \\
0.333\end{array}$ & 1.916 & 0.631 & 2.555 & 5.268 & 11.740 & $\begin{array}{c}- \\
14.095\end{array}$ & -2.425 & -0.877 & 0.007 \\
\hline 34 & API 99×KAUZ & $3 . \overline{-} 85$ & 0.333 & $0 . \overline{4} 16$ & $0 . \overline{129}$ & 2.888 & 5.268 & -1.259 & 1.188 & 15.973 & 0.082 & 0.125 \\
\hline 35 & API 99×ADANA 99 & 4.481 & $\overline{-} \cdot \overline{333}$ & 3.583 & $\begin{array}{c}- \\
0.204\end{array}$ & 7.222 & 6.268 & 8.407 & 4.024 & 17.327 & -0.310 & 0.033 \\
\hline 36 & API 99×ACSAD5 & 2.518 & 5.333 & -0.75 & 0.409 & 7.888 & 12.268 & -1.592 & -0.902 & 13.352 & 2.927 & 0.092 \\
\hline & SE(CROSSES) & 0.638 & 0.736 & 0.728 & 0.099 & 0.752 & 0.805 & 0.853 & 14.522 & 1.531 & 0.498 & 0.033 \\
\hline
\end{tabular}

$\mathrm{DH}=$ Days to $50 \%$ heading, $\mathrm{PH}=$ Plant height, $\mathrm{NS} / \mathrm{S}=$ Number of spikelets/spike, LA = leaf area, $\mathrm{T} / \mathrm{P}=\mathrm{Number}$ of tillers/plant , NS/P = Number of spikes /plant, NG/S = Number of grains/spike, BY/P= Biological yield/plant, GY/P = Grain yield/plant, TGW=1000 grain weight, $\mathrm{HI}=$ Harvest index .

\section{Number of spikes per plant (NS/P) :}

Three female lines out of twelve exhibited significant gca effects, three lines namely L12 (5.787), L3 (4.342) and L8 (1.564) showed significant positive gca effects for the trait. One tester T1 (0.703) showed significant positive gca effects. twenty two hybrids showed significant sca effects out of which twelve crosses namely T1 $\times$ L12 (18.185), T3 × L8 (17.601), $\mathrm{T} 3 \times \mathrm{L} 12(12.268), \mathrm{T} 2 \times \mathrm{L} 10(11.546), \mathrm{T} 2 \times \mathrm{L} 11$ (10.212), T1 $\times$ L3 (9.407), T3 $\times$ L11 (6.268), T2 $\times$ L8 $:$ T2 $\times$ L12 (6.212), T2 $\times$ L9 (5.879), T1 × L9 (5.851), T3 $\times$ L9 : T3 $\times$ L10 (5.268), T1 $\times$ L10 (3.851), T2 $\times$ $\mathrm{L} 2$ (3.768), $\mathrm{T} 1 \times \mathrm{L} 11$ (3.518), $\mathrm{T} 1 \times \mathrm{L} 6$ (3.185), $\mathrm{T} 2 \times$ L7 (2.546), T2 $\times$ L5 (1.879), T3 $\times$ L1 (1.712), T1 $\times$ $\mathrm{L} 2(1.407)$ and $\mathrm{T} 3 \times \mathrm{L} 4(0.935)$

\section{Number of grains per spike (NG/S):}

Six parental lines out of fifteen showed significant gca effects L8 (9.287), L6 (7.398), L2 (5.064), T3 (3.925), L3 (3.731) and L5 (0.842) in positive direction. Twenty cross combinations showed significant positive sca effects. Cross T1 $\times$ L8 (24.518) showed highest significant positive sca effects followed by T3 $\times$ L8 (23.407), T2 $\times$ L10 (15.407), $\mathrm{T} 1 \times \mathrm{L} 2(14.185), \mathrm{T} 2 \times \mathrm{L} 3: \mathrm{T} 3 \times \mathrm{L} 9$ (11.740), T3 $\times$ L7 (9.074), T3 $\times$ L11 (8.407), T1 $\times$ L1 1 (5.518), $\mathrm{T} 2 \times \mathrm{L} 11$ (4.740), $\mathrm{T} 3 \times \mathrm{L} 3$ (4.407), $\mathrm{T} 1 \times \mathrm{L} 4$ (4.296), $\mathrm{T} 2 \times \mathrm{L} 1$ (4.185), $\mathrm{T} 2 \times \mathrm{L} 2$ (3.074), $\mathrm{T} 1 \times \mathrm{L} 6$ (2.518), T2 $\times$ L12 (2.407), T1 $\times$ L5 : T3 $\times$ L6 (1.740), $\mathrm{T} 1 \times \mathrm{L} 9$ (1.518), and T1 $\times$ L1 $(0.962)$. Highest significant positive sca effects were exhibited by the cross $\mathrm{T} 1 \times \mathrm{L} 8$ identified as best general combiner for the trait and cross $\mathrm{T} 3 \times \mathrm{L} 8$ as best specific cross combination and can be useful for further improvement of the trait. Similar results were also observed by (Singh et al., 2012) during their line $x$ tester experiment in bread wheat (Triticum aestivum L.) and (Lohithaswa et al., 2013) in tetraploid wheat.

\section{Biological yield per plant (BY/P) :}

Lines L8 (23.607), L5 (20.219), L3 (12.897), L2 (9.720) and L6 (8.629) exhibited highly significant positive gca effects. twelve hybrids showed significant sca effects namely, T3 × L8 (90.624), T2 $\times$ L9 (73.432), T1 × L11 (47.476), T1 × L8 (34.894), $\mathrm{T} 1 \times \mathrm{L} 10(34.789), \mathrm{T} 2 \times \mathrm{L} 4(33.861), \mathrm{T} 3 \times \mathrm{L} 1$ (32.982), T2 $\times$ L10 (30.889), T2 $\times$ L7 (27.293), T1 $\times$ L6 (17.538), T3 $\times$ L2 (16.602) and T2 $\times$ L11 (15.075) exhibited significant positive sca effects. For biological yield, positive gca and sca effects are desirable. Therefore, L8 identified as best general combiner and T3 $\times$ L8 as the best specific cross combination in the present investigation for biological yield per plant. These results showed similarity with earlier observations of (Kumar et al., 2015) and (Nour et al., 2011) for this trait.

\section{Grain yield per plant (GY/P) :}

Seven parental L8 (18.182), L5 (14.498), L6 (13.228), L3 (9.215), L11 (3.768), T2 (1.902) and L10 (0.918) identified with significant positive gca values. Twenty two cross combinations showed significant positive sca effects. Cross T3 $\times$ L8 (48.402), $\mathrm{T} 1 \times \mathrm{L} 8$ (31.638), T2 × L9 (24.850), T2 $\times$ L10 (24.405) , T2 × L7 (19.222), T1 × L11 (19.122) , T3 $\times$ L11 (17.327) , T2 $\times$ L2 (16.037) , T3 $\times$ L10 (15.973), T2 × L11 (14.904), T2 × L8 (14.556), T3 $\times \mathrm{L} 12(13.352), \mathrm{T} 1 \times \mathrm{L} 12(12.576), \mathrm{T} 1 \times \mathrm{L} 1(11.926)$ $, \mathrm{T} 1 \times \mathrm{L} 3(9.813), \mathrm{T} 3 \times \mathrm{L} 5(8.784), \mathrm{T} 1 \times \mathrm{L} 6(8.495)$ , T1 $\times$ L4 (7.247), T3 × L7 (3.886), T1 × L2 (3.596) , T1 $\times$ L10 (2.427) and T3 $\times$ L3 (2.185). In the present investigation line L8 identified as best general combiner and among hybrids, T3 $\times$ L8 emerged as best specific combiner for grain yield. The results were in close confirmation with the earlier observation of (Esmail, 2007) and (Kapoor et al., 2011).

\section{0 grain weight (TGW) :}

Five lines showed significant gca effects, L3 (5.488), L4 (3.934), L6 (2.154), L12 (1.004) and L2 (0.793) exhibited significant positive gca effects and among testers, T1 (1.034), showed positive significant gca effects. Fourteen hybrids showed significant sca effects. T1 $\times$ L3 (7.004) showed highest significant positive sca effects followed by T3 $\times$ L6 (4.629), T1 $\times$ L12 (3.190), T1 $\times$ L2 (3.163), T3 $\times$ L12 (2.927), T2 $\times$ L5 (2.514), T2 $\times$ L8 (2.498), T1 $\times$ L4 (2.395), T2 $\times$ L9 (2.381), T3 $\times$ L7 (1.979), T3 $\times$ L1 (1.254), T2 $\times$ L12 (0.735), T2 $\times$ L2 (0.689), and T1 $\times$ L8 (0.596). 1000-grain weight is an important indirect selection criterion for the selection of grain yield thus significant gca values in positive direction indicates good general combining ability. On the basis of these result, line L3 identified as best general combining parent and cross $\mathrm{T} 1 \times \mathrm{L} 3$ was identified as best specific combination for 1000 grain weight. These results are in agreement with the earlier studies carried out by (Nour et al., 2011) and (Istipliler et al., 2015) to determine the combining abilities of wheat genotypes for yield and related traits 


\section{Harvest index (HI) :}

Line L6 (0.052), L5 (0.042), L8 (0.034) and L10 (0.022) and among testers, T3 (0.010), showed significant positive gca effects. Among the crosses, T1 $\times \mathrm{L} 8(0.146), \mathrm{T} 2 \times \mathrm{L} 10(0.125), \mathrm{T} 3 \times \mathrm{L} 10(0.125)$, $\mathrm{T} 3 \times \mathrm{L} 12(0.092), \mathrm{T} 2 \times \mathrm{L} 8(0.081), \mathrm{T} 3 \times \mathrm{L} 5(0.080)$ , T1 × L12 ) $0.078($, T3 $\times$ L7 (0.076), T2 × L11 $(0.072), \mathrm{T} 1 \times \mathrm{L} 1(0.070), \mathrm{T} 2 \times \mathrm{L} 7(0.057), \mathrm{T} 1 \times \mathrm{L} 4$ $(0.053), \mathrm{T} 2 \times \mathrm{L} 6(0.048), \mathrm{T} 1 \times \mathrm{L} 3(0.047)$ and T3 $\times$ L11 (0.033). showed significant positive sca effects. Harvest index is also an important indirect selection criterion for grain yield. L6 emerged as good general combiner and cross $\mathrm{T} 1 \times \mathrm{L} 8$ was identified as the most superior cross combination for the trait. Similar results were also reported by (Jatav et al., 2014) during their line $\times$ tester experiment for analysis of combining ability and heterosis of morphological traits in wheat. Combining ability analysis in the present investigation suggested an idea about breeding methodology to be applied and use of promising cross combinations for further wheat improvement programmers. Present investigation revealed that parents L3, L5, L6, L8 and T1 were good general combiners for most of the characters studied and should be utilized further in breeding programmers for developing superior varieties. Present findings also revealed the significance of non-additive gene action for grain yield and its contributing components. The presence of significant no additive genetic variances suggested that high performance of yield and contributing traits can be fixed in subsequent segregating generation of $\mathrm{T} 1 \times \mathrm{L} 8, \mathrm{~T} 2 \times \mathrm{L} 10, \mathrm{~T} 2 \times \mathrm{L} 11, \mathrm{~T} 1 \times \mathrm{L} 11, \mathrm{~T} 2 \times \mathrm{L} 2$ and $\mathrm{T} 3 \times$ L7 . These crosses could be the desirable choice for exercising single plant selection in advanced generations as all these crosses exhibited highly positive sca effects for various yield contributing traits.

\section{References}

Arunachalam, V.1974. The falacy behind the use of modified line $x$ tester design. Indian J. Genet., 34: 280-28.

Chaudhry, M.A., Akhtar, M.S. and Ahmad, M.T.1992.Combining ability analysis for flag leaf area, yield and its components in spring wheat. J. of Agri. Research, 30(1):17-23.

Cifci, E.A., and Yagdi, K. 2010. The research of the combining ability of agronomic traits of bread wheat in F1 and F2 generations. U. U. Ziraat Fakultesi Dergisi, 24 (2): 85-92.

Dagustu, N., 2008.Combining ability analysis in relation to heterosis for grain yield per spike and agronomic traits in bread wheat (Triticum aestivum L.).Turkish J. of Field Crops, 13(2): 4961.

Esmail, R.M., 2007. Detection of genetic components through triple test cross and line $\mathrm{x}$ tester analysis in bread wheat. World J. of Agri. Sci., 3(2):184190.

FAO. 2018. Statistical yearbook 2018 World Food and Agriculture.

FAO. 2006. Planning For the Future: An Assessment of Food Security Early Warning Systems in SubSaharan Africa-Synthesis Report, By J. Tefft, M. Mcguire \& M. Maunder.

Fellahi, Z.E.A.,Hannachi, A., Bouzerzour, H. And Boutekrabt, A. 2013. Line $\times$ Tester mating design analysis for grain yield and yield related traits in bread wheat (Triticum aestivum L.). Int. J. of Agro, volume 2013, Article ID 201851,9pages.

Gorjanovic, B., and Balalic, M.K.2004. Genetic analysis for grain weight per spike and harvest index in macaroni wheat. Genetika , 36 (1) : 23 29.

Hassan,G., Mohammad, F., Afridi, S.S. and Khalil, I. 2007. Combining ability in the F1 generations and yield components in Wheat.Sarhad.J.Agric.,23(4):937-942.

Istipliler, D., Ilker, E., Tonk, F.A., Civi, G.and Tosun, M.2015. Line $\mathrm{x}$ Tester analysis and estimating combining abilities for yield and some yield components in bread wheat .Turkish J.of Field Crops, 20(1):72-77.

Jain,S.K., and Sastry, E.V.D. 2012.Heterosis and combining ability for grain yield and its contributing traits in bread wheat (Triticum aestivum L.). J. of Agri. and Allied Sci., 1(1): 1722.

Jatav, M., Jatav, S.K. and Kandalkar , V.S.2014.Combining ability and heterosis analysis of morpho-physiological characters in wheat. Annals of Plant and Soil Res., 16(2): 79-83.

Kapoor ,E., Mondal, S.K. and Dey,T .2011. Combining ability analysis for yield and yield contributing traits in winter and spring wheat combinations. J.Wheat Res.3(1):52-58.

Kempthorne, O., 1957. An introduction to statistics. John Willey and Sons Inc. New York, pp. 468-471.

Kumar, A., Harshwardhan, Kumar, A. and Prasad, B. 2015. Combining ability and gene interaction study for yield, its attributing traits and quality in common wheat. J. of Applied and Nat. Sci. 7 (2):927-934.

Kumar,A., Prasad, B.and Kumar, A.2015. Study of variance component of combining ability for yield and its attributing traits in bread wheat.Elixir Agriculture, 81, 31425 - 31426.

Morris, R. and Sears, E. R., 1967. The cytogenetics of wheat and its relatives. In : Quisenberry and Reitz (Eds), Wheat and Wheat Improvement, Madison, USA, pp. 19-87.

Nour, A., Nadya, A.R., El-Fateh, H.S.A. and Mostafa, A.K. 2011. Line $x$ Tester analysis for yield and its traits in bread wheat. Egypt. J. Agric. Res., 89(3): 979-990. 
Panse, V.G., and Sukhatme, P.V. 1967. Statistical Methods of Agricultural Workers. 2nd Endorsement, ICAR Publication, New Delhi, India, pp-381.

Poehlman, J.M., and Sleeper, D.A. 1995. Breeding Field Crops. $4^{\text {th }}$ edition. ANI, INC. Westport, Connecticut.

Prasad, B., 2014. Heterotic vigour studies in forage sorghum hybrid by multiple criteria. J. of Hill Agri., 5 (2): 182-185.

Raj, P., and Kandalkar, V.S. 2013. Combining ability and heterosis analysis for grain yield and its components in wheat. J. of Wheat Res., 5(1): 4549.

Shabbir, G., Kiran, T., Akram, Z., Tabassum, M.I. and Shah, K.N. (2012 Genetics of some biometric traits in bread wheat (triticum aestivum L.). J. Agric. Res., 50(4): 457-468.
Singh A., Kumar A., Ekhlaque Ahmad, Swati and Jaiswal, J.P. 2012.Combining ability and gene action studies for seed yield, its components and quality traits in bread wheat (Triticum aestivum L. em Thell.) Electronic Journal of Plant Breeding , 3 (4) : $964-972$.

Vanpariya, L.G., Chovatia,V.P. and Mehta, D.R. 2006. Heterosis for grain yield and its attributes in bread wheat (Triticum aestivum L.). Nat. J. of Plant Imp., 8(2):100-102.

Zeeshan, M., Arshad, W., Ali, S. Tariq, M. and Siddique, M. 2013. Estimation of combining ability effects for some yield related metric traits in intra-specific crosses among different spring wheat (Triticum aestivum L.) genotypes. Int. J. of Advanced Res., 1(3): 6-10 .

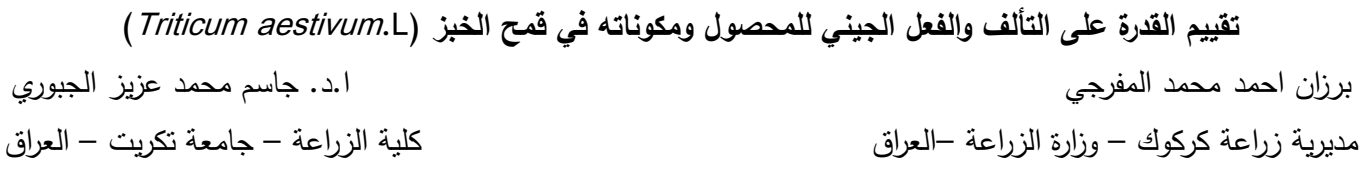

تم دراسة تحليل القدرة على التالف والفعل الجيني على محصول الحبوب ومكوناته في قمح الخبز باستخدام اثثي عثر سلالة (FLORKA، (5ACSAD , 99 ADANA، KAUZ ، OASIS ، ALLAI ، HIDHAB ، SITEMALL ، ARAS ، RIZGARY ، KLAK ، MALLIN وثلاثة كثافات ( SHAM،ABU GHRAIB 3 و 6 SH API) ، لإنتاج 36 هجين فردي في تصميم التزاوج السلالة ، الكثاف والتي نم تقييمها في تصميم القطاعات العشوائية الكاملة في ثلاث مكررات. اظهرت دراسة الفعل الجيني ناثير الفعل الجيني غير الاضافي على جميع الصفات ،

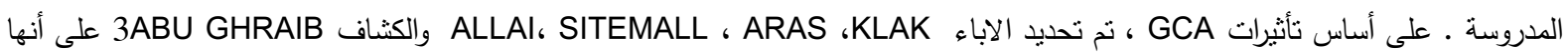
ذات قدرة عامة جيدة على التالف. كما وجد ان الهجن ABU GHRAIB 3 × ALLAI و SHAM 6 × KAUZ كانا ذو قدرة خاصة على التالف

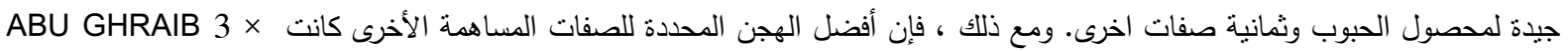

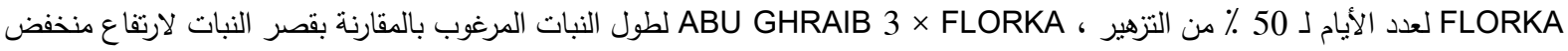

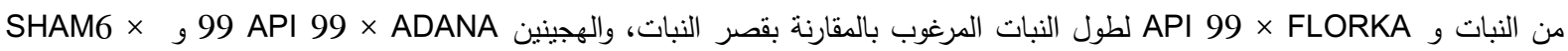
FLORK بالنسبة لعدد السنيبلات للسنبلة الواحدة، SHAM6× KAUZ لمساحة الورقة، 5ABU GHRAIB 3 × ACSAD لعدد الافرع الكلية

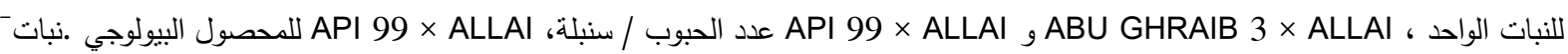

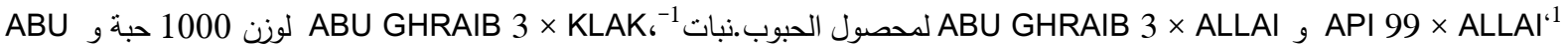

